\title{
Applications of gyrator transform for image processing
}

\author{
José A. Rodrigo *, Tatiana Alieva, María L. Calvo \\ Universidad Complutense de Madrid, Facultad de Ciencias Físicas, Ciudad Universitaria s/n, Madrid 28040, Spain
}

Received 10 May 2007; received in revised form 8 June 2007; accepted 11 June 2007

\begin{abstract}
Gyrator transform is a new tool for manipulation of two-dimensional signals such as images or laser beam profiles. Here we demonstrate various applications of the gyrator transform for image processing. Several aspects such as noise reduction, filtering and encryption in the gyrator domains are discussed. These operations can be performed by numerical calculations or by an appropriate optical set up. (C) 2007 Elsevier B.V. All rights reserved.
\end{abstract}

PACS: 42.30.-d

Keywords: Gyrator transform; Image filtering; Encryption

\section{Introduction}

The first-order optical systems, mathematically described by canonical integral transforms, remain the basic elements for analogue optical information processing and beam transformation. Several new applications of these systems have been proposed in the past decade. In particular the first-order optical systems, performing the fractional Fourier transform, have been used for shift-variant filtering, noise reduction, encryption, etc. [1]. Another ones have served as mode converters. The Hermite-Gaussian (HG) beam passing through them are converted into the helicoidal vortex Laguerre-Gaussian (LG) mode or other stable modes carrying the fractional topological charge [2,3]. The generalized mode converter can be described by the gyrator transform (GT), called in [4] as a cross-gyrator. The GT as well as the fractional FT belong to the orthosymplectic class of the linear canonical integral transforms $[4,7]$ and describe the rotations in the phase space. In the contrast

\footnotetext{
* Corresponding author. Tel.: +34 913944678.

E-mail address: jarmar@fis.ucm.es (J.A. Rodrigo).
}

to the fractional Fourier transform (see for example $[1,5]$ and references there in), the gyrator operation is not widley known. Recently we have derived the main properties of the gyrator transform [6] and designed the flexible optical set up which performs this transform $[7,8]$. The purpose of this paper is to discuss the applications of the GT for image processing. The paper is organized as following. In the Section 2 we introduce the gyrator transform and briefly mention its main properties. The Section 3 is dedicated to the filtering in the gyrator domain. In the Section 4 we consider its application for noise reduction. The application of the GT for encryption of two dimensional signals is discussed in the Section 5, followed by concluding remarks.

\section{Fundamentals}

Gyrator operation is mathematically defined as a linear canonical integral transform which produces the rotation in twisted position-spatial frequency planes $\left(x, q_{y}\right)$ and $\left(y, q_{x}\right)$ of phase space [4]. Thus, the gyrator transform at parameter $\alpha$, called further as a rotation angle, of a two-dimensional function $f_{\mathrm{i}}\left(\mathbf{r}_{\mathrm{i}}\right)$ is written in the following form: 


$$
\begin{aligned}
f_{\mathrm{o}}\left(\mathbf{r}_{\mathrm{o}}\right)= & R^{\alpha}\left[f_{\mathrm{i}}\left(\mathbf{r}_{\mathrm{i}}\right)\right]\left(\mathbf{r}_{\mathrm{o}}\right) \\
= & \iint f_{\mathrm{i}}\left(x_{\mathrm{i}}, y_{\mathrm{i}}\right) K_{\alpha}\left(x_{\mathrm{i}}, y_{\mathrm{i}}, x_{\mathrm{o}}, y_{\mathrm{o}}\right) \mathrm{d} x_{\mathrm{i}} \mathrm{d} y_{\mathrm{i}} \\
= & \frac{1}{|\sin \alpha|} \times \iint f_{\mathrm{i}}\left(x_{\mathrm{i}}, y_{\mathrm{i}}\right) \\
& \times \exp \left(\mathrm{i} 2 \pi \frac{\left(x_{\mathrm{o}} y_{\mathrm{o}}+x_{\mathrm{i}} y_{\mathrm{i}}\right) \cos \alpha-\left(x_{\mathrm{i}} y_{\mathrm{o}}+x_{\mathrm{o}} y_{\mathrm{i}}\right)}{\sin \alpha}\right) \mathrm{d} x_{\mathrm{i}} \mathrm{d} y_{\mathrm{i}},
\end{aligned}
$$

where $\mathbf{r}_{\mathrm{i}, \mathrm{o}}^{t}=\left(x_{\mathrm{i}, \mathrm{o}}, y_{\mathrm{i}, \mathrm{o}}\right)$ indicates the input and output coordinates, respectively. Notice that $t$ stands for transposition operation.

For $\alpha=0$ it corresponds to the identity transform, for $\alpha=\pi / 2$ it reduces to the direct/inverse Fourier transform with rotation of the coordinates at $\pi / 2$, for $\alpha=\pi$ the reverse transform described by the kernel $\delta\left(\mathbf{r}_{\mathrm{o}}+\mathbf{r}_{\mathrm{i}}\right)$ is obtained. The GT is periodic and additive with respect to parameter $\alpha: R^{\alpha} R^{\beta}=R^{\alpha+\beta}$. The inverse GT corresponds to the GT at angle $-\alpha$. Note that in spite of a certain similarity between the fractional FT and the GT they do not coincide since the kernel of the fractional FT is a product of the spherical and plane waves. Meanwhile the kernel of the GT is a product of hyperbolic and plane waves.

The main theorems for the GT derived in [6] are displayed in Table 1, where the following notations are used. In the first and the second lines the vectors $\mathbf{v}^{t}=\left(v_{x}, v_{y}\right)$, $\tilde{\mathbf{v}}^{t}=\left(v_{y}, v_{x}\right)$ and $\mathbf{k}^{t}=\left(k_{x}, k_{y}\right), \tilde{\mathbf{k}}^{t}=\left(k_{y}, k_{x}\right)$ correspondingly are introduced. In the third line parameters $\sigma_{\alpha}, \sigma_{\beta}, \mathbf{S}, \beta$ are defined as:

$\sigma_{\alpha}=\operatorname{sgn}(\sin \alpha), \quad \sigma_{\beta}=\operatorname{sgn}(\sin \beta)$,

where sgn is the signum function.

$\mathbf{S}=\left(\begin{array}{cc}s_{x} & 0 \\ 0 & s_{y}\end{array}\right)$ and $\cot \beta=\frac{\cot \alpha}{s_{x} s_{y}}$.

Note that $s_{x}$ and $s_{y}$ are real numbers.

Based on the matrix formalism for the first-order lossless optical systems, it has been recently shown [7] that the GT for the large range of angles $\alpha$ can be realized by flexible optical system which contains only three generalized lenses with fixed distances (free-space intervals) between them. The angle $\alpha$ is changed by rotation of the

Table 1

Main properties of the gyrator transform

Shift theorem

$R^{\alpha}\left[f_{\mathrm{i}}\left(\mathbf{r}_{\mathrm{i}}-\mathbf{v}\right)\right]\left(\mathbf{r}_{\mathrm{o}}\right)=$

$\exp \left(\mathrm{i} \pi\left(v_{x} v_{y} \sin 2 \alpha-2 \mathbf{r}_{\mathrm{o}}^{t} \tilde{\mathbf{v}} \sin \alpha\right)\right) R^{\alpha}\left[f_{\mathrm{i}}\left(\mathbf{r}_{\mathrm{i}}\right)\right]\left(\mathbf{r}_{\mathrm{o}}-\mathbf{v} \cos \alpha\right)$

Plane wave modulation

$R^{\alpha}\left[f_{\mathrm{i}}\left(\mathbf{r}_{\mathrm{i}}\right) \exp \left(-\mathrm{i} 2 \pi \mathbf{k}^{t} \mathbf{r}_{\mathrm{i}}\right)\right]\left(\mathbf{r}_{\mathrm{o}}\right)=$

$\exp \left(-\mathrm{i} \pi\left(k_{x} k_{y} \sin 2 \alpha+2 \mathbf{k}^{t} \mathbf{r}_{\mathrm{o}} \cos \alpha\right)\right) R^{\alpha}\left[f_{\mathrm{i}}\left(\mathbf{r}_{\mathrm{i}}\right)\right]\left(\mathbf{r}_{\mathrm{o}}+\tilde{\mathbf{k}} \sin \alpha\right)$

Scaling theorem

$R^{\alpha}\left[f_{\mathrm{i}}\left(\mathbf{S r}_{\mathrm{i}}\right)\right]\left(\mathbf{r}_{\mathrm{o}}\right)=$

$$
\frac{\sigma_{\beta} \cos \beta}{\sigma_{\alpha} \cos \alpha} \exp \left(\mathrm{i} 2 \pi x_{\mathrm{o}} y_{\mathrm{o}}\left(1-\left(\frac{\cos \beta}{\cos \alpha}\right)^{2}\right) \cot \alpha\right) R^{\beta}\left[f_{\mathrm{i}}\left(\mathbf{r}_{\mathrm{i}}\right)\right]\left(\frac{\cos \beta}{\cos \alpha} \mathbf{S r}_{\mathrm{o}}\right)
$$

Parseval relation

$\int R^{\alpha}\left[f_{\mathrm{i}}\left(\mathbf{r}_{\mathrm{i}}\right)\right]\left(\mathbf{r}_{\mathrm{o}}\right)\left(R^{\alpha}\left[g_{\mathrm{i}}\left(\mathbf{r}_{e}\right)\right]\left(\mathbf{r}_{\mathrm{o}}\right)\right)^{*} d \mathbf{r}_{\mathrm{o}}=\int f_{\mathrm{i}}\left(\mathbf{r}_{\mathrm{i}}\right) g_{\mathrm{i}}\left(\mathbf{r}_{\mathrm{i}}\right)^{*} d \mathbf{r}_{\mathrm{i}}$ cylindrical lenses which form the generalized lenses. Other possibility is to use the spatial light modulator for variable lens performance. Explicit equations for these generalized lenses as a function of the transformation angle $\alpha$ can be found in $[7,8]$. The experimental realization of the GT has been considered in [8]. In particular the transformation of the Hermite-Gaussian modes into other stable modes including the Laguerre-Gaussian ones obtained by this optical setup demonstrates a good agreement with theoretical prediction.

The numerical algorithm for the calculation of the GT has the same complexity as the one for the fractional FT. In this paper we use the approach based on the numerical simulation of the optical setup associated to the GT $[7,8]$. As it has been mentioned above this set up is constructed by three generalized lenses with fixed distances between them. Fresnel diffraction in the free space intervals is simulated applying the spectrum propagation method. The generalized lens action is described by the appropriate phase modulation. The test image (input function $\left.f_{\mathrm{i}}\left(x_{\mathrm{i}}, y_{\mathrm{i}}\right)\right)$ corresponds to Lena picture of $256 \times 256$ pixels embedded on a square empty matrix with $1024 \times 1024$ points (numerical window size).

\section{Filtering in gyrator domain}

One of the main goals of the signal processing is to recover useful information about an object from the related signal. The encoded information may be partially localized or spread over the entire signal domain. It may be represented by a specific pattern, which has to be detected, or may be hidden due to the noise which has to be eliminated. Therefore the signal processing methods need to be adjusted to the specific tasks and signal characteristics.

In order to include the GT in the list of the signal processing tools we have to understand its actions. As it is observed from Eq. (1), the GT, at least in its present form, can be applied only for two-dimensional signals. Therefore here we will analyze the GT of two-dimensional real signals (images).

Since the kernel of the GT is a complex function we first need to analyze the information about the image contained in the phase and the amplitude of the GT at different angles. The angle range can be reduced to $[0, \pi / 2]$ since for the real signals $f_{\mathrm{i}}\left(\mathbf{r}_{\mathrm{i}}\right)$ we have the following relations:

$$
\begin{aligned}
& R^{-\alpha}\left[f_{\mathrm{i}}\left(\mathbf{r}_{\mathrm{i}}\right)\right]\left(\mathbf{r}_{\mathrm{o}}\right)=\left[R^{\alpha}\left[f_{\mathrm{i}}\left(\mathbf{r}_{\mathrm{i}}\right)\right]\left(\mathbf{r}_{\mathrm{o}}\right)\right]^{*}, \\
& R^{\pi-\alpha}\left[f_{\mathrm{i}}\left(\mathbf{r}_{\mathrm{i}}\right)\right]\left(\mathbf{r}_{\mathrm{o}}\right)=\left[R^{\alpha}\left[f_{\mathrm{i}}\left(\mathbf{r}_{\mathrm{i}}\right)\right]\left(-\mathbf{r}_{\mathrm{o}}\right)\right]^{*} .
\end{aligned}
$$

The GT at $\alpha=0$ and at $\alpha=\pi / 2$ will be further mentioned as image and Fourier domains respectively.

Let us consider the reconstruction of the test image Lena from only phase or only amplitude of its GT for different angles $\alpha$. Thus if we introduce the following notation:

$R^{\alpha}\left[f_{\mathrm{i}}\left(\mathbf{r}_{\mathrm{i}}\right)\right]\left(\mathbf{r}_{\mathrm{o}}\right)=F_{\alpha}\left(\mathbf{r}_{\mathrm{o}}\right) \exp \left(\mathrm{i} \varphi_{\alpha}\left(\mathbf{r}_{\mathrm{o}}\right)\right)$, 
where $F_{\alpha}\left(\mathbf{r}_{\mathrm{o}}\right)>0$ is the amplitude, and $\varphi_{\alpha}\left(\mathbf{r}_{\mathrm{o}}\right)$ is the phase of the GT at angle $\alpha$, the considered operations are expressed as $R^{-\alpha}\left[\exp \left(\mathrm{i} \varphi_{\alpha}\left(\mathbf{r}_{\mathrm{o}}\right)\right)\right](\mathbf{r})$ and $R^{-\alpha}\left[F_{\alpha}\left(\mathbf{r}_{\mathrm{o}}\right)\right](\mathbf{r})$.

In Fig. 1 the amplitudes of the image reconstructed from the phase-only data (a-d) and the amplitude-only data $(\mathrm{e}-\mathrm{h})$ of the GT of the Lena image are displayed for different values of the transformation angle $\alpha$ : (a, e) $\alpha=2^{\circ},(\mathrm{b}, \mathrm{f})$ $\alpha=10^{\circ},(\mathrm{c}, \mathrm{g}) \alpha=60^{\circ},(\mathrm{d}, \mathrm{h}) \alpha=90^{\circ}$. We observe that the amplitude of the GT transform contains essential information about the structure of the image only for relatively small parameter $\alpha$. Meanwhile the GT phase preserves the information about the image edges almost for all range of $\alpha$. To this respect, the gyrator transform is similar to the fractional Fourier transform $[9,10]$. This was expected from Eq. (4) and the fact that the GT can be represented as a rotation at angle $-\pi / 4$ antisymmetric fractional FT of the rotated at $\pi / 4$ original signal [11]. Thus we can conclude that the phase information of the GT is more revelant than the amplitude one, where we exclude rather exotic images whose GT are real for a certain angle $\varphi_{\alpha}\left(\mathbf{r}_{\mathrm{o}}\right)=0$. This result underlines that the phase only filters can be applied for pattern detection and recognition in the gyrator domain. It has a particular significance for analogue optical implementation of the filtering in the gyrator domain.

The next example serves to analyze how the reconstructed image changes if we apply the same filter but in the different gyrator domains. We have chosen two binary amplitude filters of circular form which correspond to high pass $(\mathrm{H})$ and low pass $(\mathrm{L})$ filters if they are located in the Fourier domain $(\alpha=\pi / 2)$. The exact form of $\mathrm{H}$ and L filters can be seen in Fig. 2a and Fig. 3a where results of the filtering in the image domain are displayed. In general the
Figs. 2 and 3 represent the amplitude of the image filtered in the $\alpha-\mathrm{GT}$ domain, $A\left(\mathbf{r}_{\mathrm{o}}\right)$,

$A\left(\mathbf{r}_{\mathrm{o}}\right)=\left|R^{-\alpha}\left[G(\mathbf{r}) R^{\alpha}\left[f_{\mathrm{i}}\left(\mathbf{r}_{\mathrm{i}}\right)(\mathbf{r})\right]\right]\left(\mathbf{r}_{\mathrm{o}}\right)\right|$,

where $G(\mathbf{r})$ is a filter function for $\mathrm{H}$ (Fig. 2) or L (Fig. 3) filters, correspondingly. We observe that filtering is not shift invariant if angle $\alpha$ differs from $\pi / 2$.

The application of the H-filter in the GT domain leads to the local high frequency pass filtering. The area of its action depends on the filter size and the parameter $\alpha$. In particular such type of filters can be used for selective edge enhancement.

The L-filter (Fig. 3) produces image cutting together with smoothing. The area of image limitation and the degree of smoothing are enlarged with increasing of angle $\alpha$.

The purpose of the choice of these simple filters is to demonstrate the tendency of the filtering procedure in the GT domain as a function of the parameter $\alpha$. More sophisticated filters have to be design for a concrete image processing task.

\section{Hyperbolic noise reduction}

The noise reduction procedure generally consists in the localization of the noise by the application of the appropriate signal transformation with its further elimination or at least reduction and the performance of the inverse transformation. In the optimized cases the position of noise localization does not overlap with the signal. Thus for example the plane waves (or more general periodic noise) are localized in the Fourier domain. Meanwhile the spherical waves



a



e

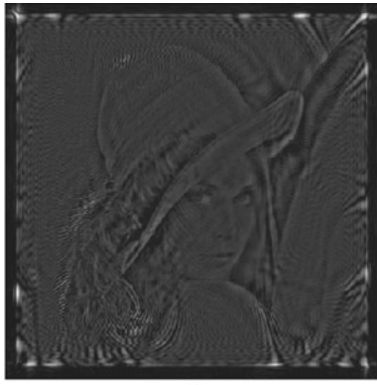

b



f

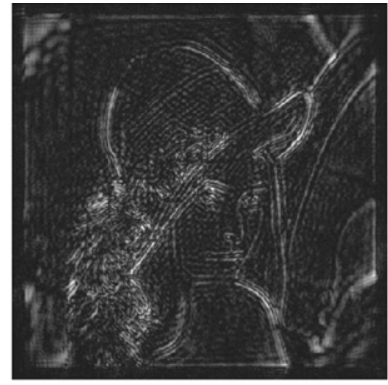

C

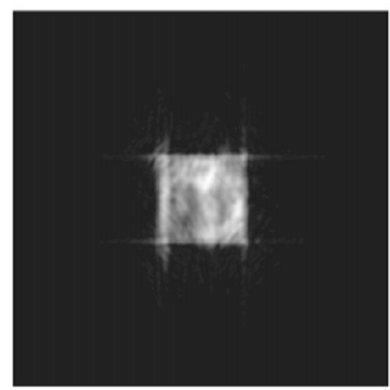

g

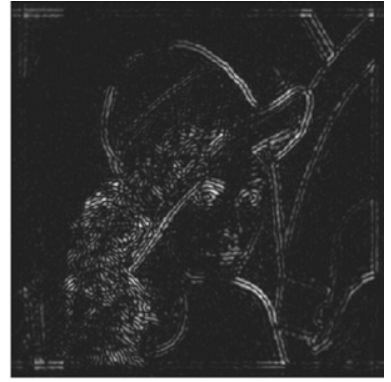

d

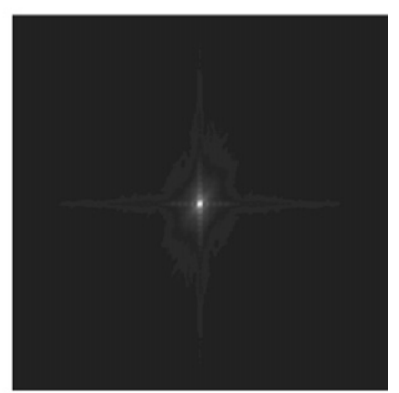

h

Fig. 1. Amplitude of the image reconstructed from the phase-only data (a-d) and the amplitude-only data (e-h) of the GT of the Lena image for different values of the transformation angle $\alpha$ : (a, e) $\alpha=2^{\circ},(\mathrm{b}, \mathrm{f}) \alpha=10^{\circ},(\mathrm{c}, \mathrm{g}) \alpha=60^{\circ},(\mathrm{d}, \mathrm{h}) \alpha=90^{\circ}$. 


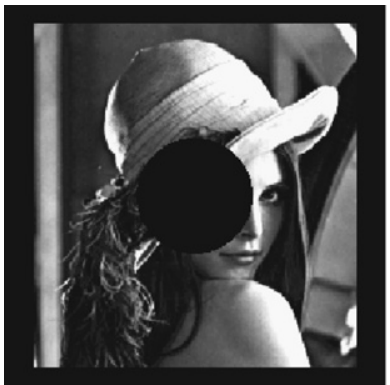

a

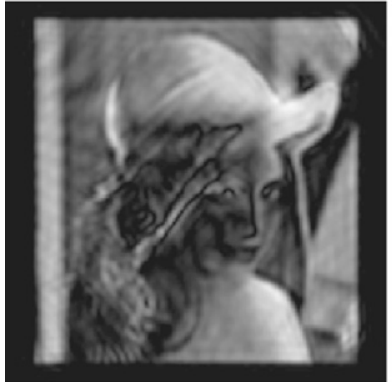

d

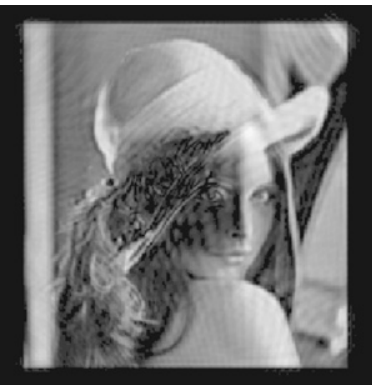

b

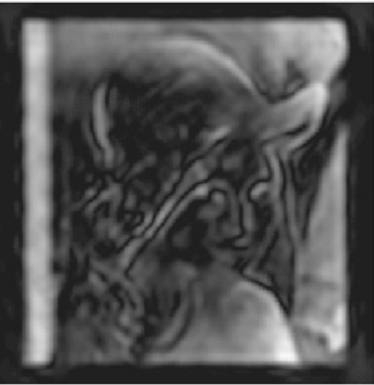

e

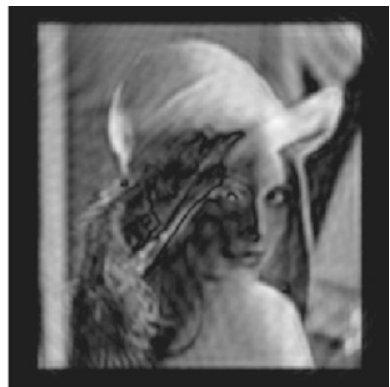

C

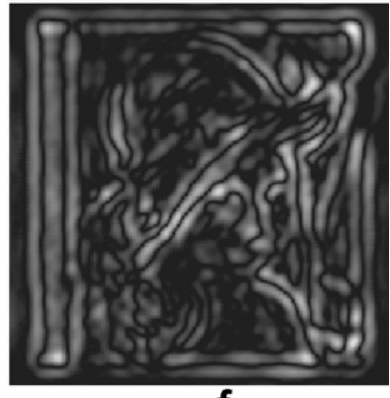

f

Fig. 2. Image sequence corresponding to the $\mathrm{H}$-filtering in the gyrator domains for the angles $\alpha=0^{\circ}, 20^{\circ}, 35^{\circ}, 45^{\circ}, 65^{\circ}$ and $90^{\circ}$ (a-f), respectively.

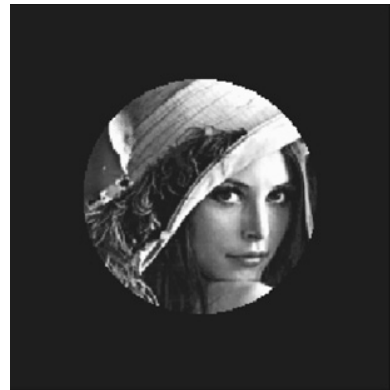

a

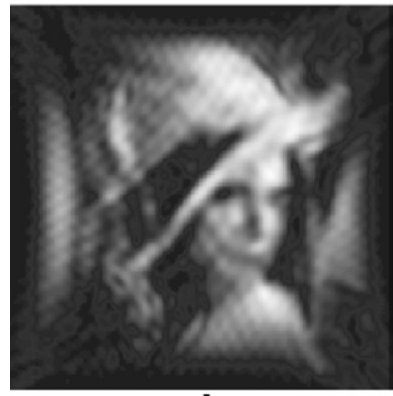

d

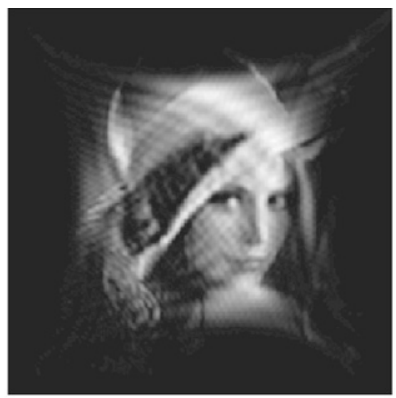

b

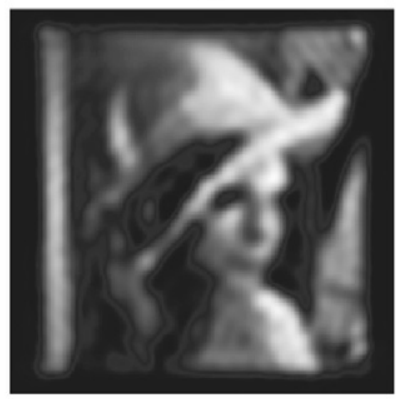

$\mathbf{e}$

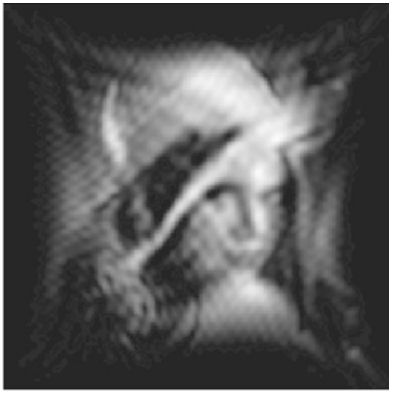

C

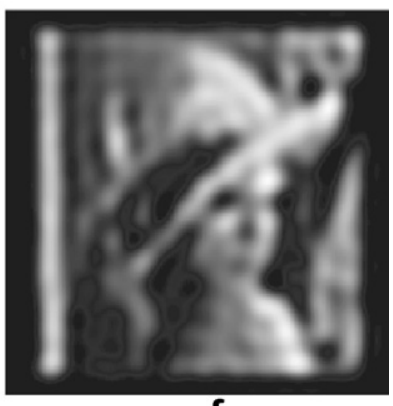

f

Fig. 3. Image sequence corresponding to the L- filtering in the gyrator domains for the angles $\alpha=0^{\circ}, 20^{\circ}, 35^{\circ}, 45^{\circ}, 65^{\circ}$ and $90^{\circ}$ (a-f), respectively.

(also known as chirp functions) can be localized in the appropriated fractional Fourier domains [1].

Let us demonstrate that the additive hyperbolic type noise, expressed by $A \cos (2 \pi c x y)=A(\exp (-\mathrm{i} 2 \pi c x y)+$ $\exp (\mathrm{i} 2 \pi c x y)) / 2$ can be removed by the filtering in the corresponding gyrator domains. The test image $s(x, y)$ corrupted by the additive hyperbolic noise:

$f(x, y)=s(x, y)+A \cos \left(2 \pi c\left(x-v_{x}\right)\left(y-v_{y}\right)\right)$,

is represented in Fig. $4 \mathrm{a}$.
It is easy to see from the shift theorem (Table 1) that the GT of the hyperbolic wave $\exp \left(\mathrm{i} 2 \pi c\left(x-v_{x}\right)\left(y-v_{y}\right)\right)$ becomes the Dirac delta function for $c=\cot \alpha$ :

$$
\begin{aligned}
& R^{\mp \alpha}\left[\exp \left( \pm \mathrm{i} 2 \pi \cot \alpha\left(x-v_{x}\right)\left(y-v_{y}\right)\right)\right]\left(\mathbf{r}_{\mathrm{o}}\right) \\
& \quad=\delta\left(\mathbf{r}_{\mathrm{o}} \pm \mathbf{v} \cos \alpha\right) \exp \left( \pm \mathrm{i} \pi v_{x} v_{y} \sin 2 \alpha\right),
\end{aligned}
$$

where $\mathbf{v}^{t}=\left(v_{x}, v_{y}\right)$ is a spatial shift. Thus analyzing the gyrator spectra (the squared moduli of the GT) of $f(x, y)$ at angles $\pm \alpha$ we observe the peaks ( $\delta$ functions) corre- 
a

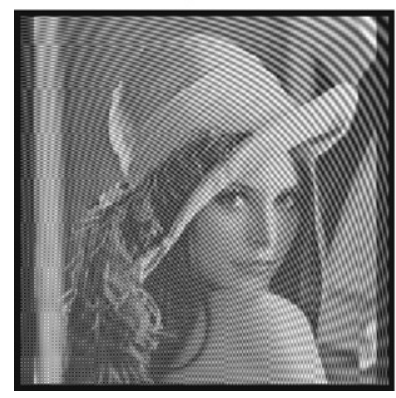

b

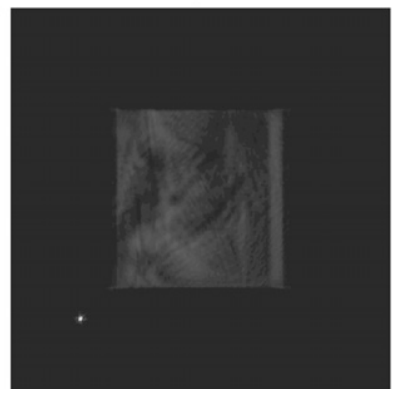

C

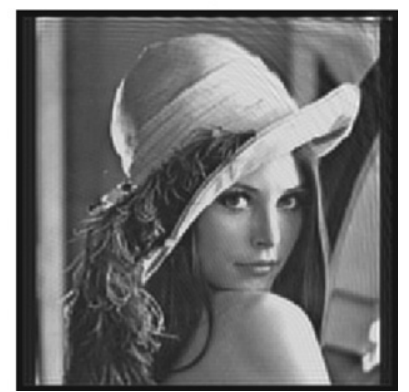

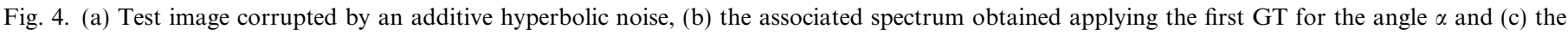
denoised image.

sponding to the hyperbolic noise. These terms can be eliminated by a simple blocking mask. After that the signal is recovered by applying the inverse gyrator transform.

Applying the GT at angle $\alpha=\arctan (1 / c)=10 \pi / 9$ to the test image its spectrum is obtained, Fig. $4 \mathrm{~b}$. The noise is localized in the white spot area associated to the function $\delta\left(\mathbf{r}_{\mathrm{o}}+\mathbf{v} \cos \alpha\right)$, (9). After application of blocking mask in this area, the GT at angle $-2 \alpha$ is performed where we again observe the noise localization associated to the function $\delta\left(\mathbf{r}_{\mathrm{o}}-\mathbf{v} \cos \alpha\right)$. Repeating the filtering procedure and applying the GT at $\alpha$ the input signal without noise is finally recovered, Fig. 4c. One can appreciate the significant improvement of the final image with respect to the one corrupted by the noise (compared with Fig. 4a). Therefore the filtering procedure requires performing three gyrator transforms (at angles $\alpha,-2 \alpha$ and $\alpha$ ) and two blocking mask applications in order to eliminate the noise components $\exp \left( \pm \mathrm{i} 2 \pi c\left(x-v_{x}\right)\left(y-v_{y}\right)\right)$.

\section{Image encryption in gyrator domains}

Recently many algorithms for optical image encryption based on the random phase masks filtering in the fractional Fourier transforms have been proposed [12,13]. The main advantage of these algorithms is that for the correct image decryption we have to know the random phase masks together with the corresponding fractional FT domains where they have been located during the encryption proce- dure. On the other words, the fractional domains also play the role of encoding parameters. Based on a certain similarity between the fractional FT and the GT the proposed techniques can be expanded on the gyrator domains.

Let us consider the following encryption/decryption scheme. The encryption procedure consists in a cascade of $N$ operations: gyrator transform at angle $\alpha_{n}$ with further result multiplication at random phase mask $\exp \left(\mathrm{i} \phi_{n}\right)$ for $n=1,2 \ldots, N$ which can be summarized as:

$F=\exp \left(\mathrm{i} \phi_{N}\right) R^{\alpha_{N}}\left[\ldots\left[\exp \left(\mathrm{i} \phi_{2}\right) R^{\alpha_{2}}\left[\exp \left(\mathrm{i} \phi_{1}\right) R^{\alpha_{1}}[f]\right]\right]\right]$.

The decryption procedure is written correspondingly as: $f=\exp \left(-i \phi_{1}\right) R^{-\alpha_{1}}\left[\ldots\left[\exp \left(-i \phi_{N-1}\right) R^{-\alpha_{N-1}}\left[\exp \left(-i \phi_{N}\right) R^{-\alpha_{N}}[F]\right]\right]\right]$.

This encryption/decryption procedure for the cascade of two steps has been applied to the Lena image. One of the corresponding random phase mask (key) is shown in Fig. 5a. The encrypted image in two gyrator domains for $\alpha_{1}=100^{\circ}, \alpha_{2}=10^{\circ}$ is displayed in Fig. 5b. The decryption with the correct random phase masks and the inverse transforms for right keys $\alpha_{1}, \alpha_{2}$ leads to the reconstruction of the original image Fig. 5c. Meanwhile the reconstruction with the correct random phase masks but wrong keys produces unrecognizable image, Fig. 5d.

Similar results (not displyed here for briefty) are obtained for other transformation angles $\left(\alpha_{1}, \alpha_{2}\right)$ demostrating the GT feasibility for encryption techniques. a

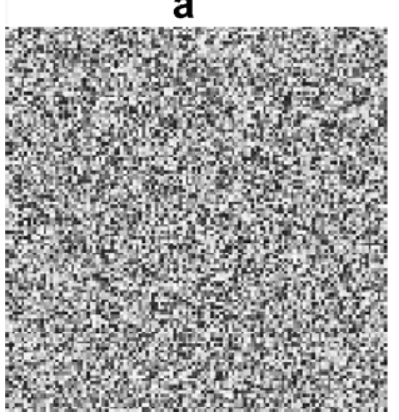

b



C

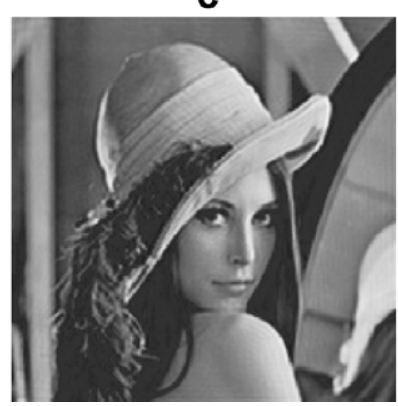

d



Fig. 5. A random phase mask (key), encrypted image in two gyrator domains (for $\alpha_{1}=100^{\circ}, \alpha_{2}=10^{\circ}$ ), and the image reconstruction applying the correct and wrong key are displayed in $(\mathrm{a}-\mathrm{d})$, respectively. 


\section{Conclusion}

In this paper we have demonstrated the main peculiarities of the two-dimensional signal processing in the gyrator transform domain. The applications of the gyrator transform for hyperbolic noise reduction and image encryption have been proposed. The filtering in GT domains demonstrated here has an illustrative character. Certainly, more sophisticated filters have to be developed for every particular case and a further research has to be done in order to make a comparison to other available image processing tools. The GT can be implemented by a rather simple optical scheme $[7,8]$ or calculated numerically. This opens perspectives for its potential applications in optical and digital information processing.

\section{Acknowledgement}

Spanish Ministry of Education and Science is acknowledged for financial support, project TEC 2005-02180/MIC.

\section{References}

[1] H.M. Ozaktas, Z. Zalevsky, M. Alper Kutay, The Fractional Fourier Transform with Applications in Optics and Signal Processing, John Wiley, NY, USA, 2001.

[2] E.G. Abramochkin, V.G. Volostnikov, J. Opt. A: Pure Appl. Opt. 6 (2004) S157.

[3] M.W. Beijersbergen, L. Allen, H.E.L.O. Van der Veen, J.P. Woerdman, Opt. Commun. 96 (1993) 123.

[4] R. Simon, K.B. Wolf, J. Opt. Soc. Am. A 17 (2000) 342.

[5] T. Alieva, M.J. Bastiaans, M.L. Calvo, EURASIP J. Appl. Signal Process 2005 (2005) 1498.

[6] J.A. Rodrigo, T. Alieva, M.L. Calvo, Opt. Express 15 (2007) 2190.

[7] J.A. Rodrigo, T. Alieva, M.L. Calvo, J. Opt. Soc. Am. A 23 (2006) 2494.

[8] J.A. Rodrigo, T. Alieva, M.L. Calvo, J. Opt. Soc. Am. A, in press.

[9] T. Alieva, M.L. Calvo, J. Opt. Soc. Am. A 20 (2003) 533.

[10] T. Alieva, M.L. Calvo, Opt. Spectrosc. 95 (2003) 110.

[11] M. Bastiaans, T. Alieva, J. Opt. Soc. Am. A 23 (2006) 1875.

[12] G. Unnikrishnan, J. Joseph, K. Singh, Opt. Lett. 25 (2000) 887.

[13] B. Zhu, S. Liu, Q. Ran, Opt. Lett. 25 (2000) 1159. 